\title{
Does Emergency Medical Services Transportation Mitigate Post-stroke Discharge Disability? A Prospective Observational Study
}

\author{
Sudha Xirasagar, MBBS, $P h D^{\top}{ }^{1}$, Yuqi Wu, MPH, MPA ${ }^{7}$, Khosrow Heidari, $M S, M A^{2}$, \\ Jiera Zhou, $P h D^{3}$, Meng-han Tsai, $P h D^{4}$, James W. Hardin, $P h D^{5}$, Robert Wronski, $M B A^{6}$, \\ Dana Hurley, Pharm. $D^{7}$, Edward C. Jauch, MD, MS, FAHA, FACEP', and Souvik Sen, \\ $\mathrm{MD}, \mathrm{MPH}^{9}$
}

\begin{abstract}
'Department of Health Services Policy and Management, Arnold School of Public Health, University of South Carolina, Columbia, SC, USA; ${ }^{2}$ South Carolina Department of Health and Environmental Control, Columbia, SC, USA; ${ }^{3}$ Statistics Collaborative, Inc., Washington, DC, USA; ${ }^{4}$ Department of Health, Human Services and Public Policy, California State University-Monterey Bay, Seaside, CA, USA; ${ }^{5}$ Department of Epidemiology and Biostatistics, Arnold School of Public Health, University of South Carolina, Columbia, SC, USA; BBureau of Emergency Medical Services, South Carolina Department of Health and Environmental Control, Columbia, SC, USA; ${ }^{7}$ Genentech, Inc., South San Francisco, CA, USA; ${ }^{8} \mathrm{HCA}$ Healthcare, Mission Research Institute, Asheville, NC, USA; ${ }^{9}$ Prisma Health Stroke Unit, Dept of Neurology, University of South Carolina School of Medicine, Columbia, SC, USA.
\end{abstract}

BACKGROUND: Whether emergency medical services (EMS) transport improves disability outcomes compared with other transport among acute ischemic stroke (AIS) patients is unknown.

OBJECTIVE: To study severity-adjusted associations of hospital arrival mode (EMS vs. other transport) with inhospital and discharge disability outcomes.

DESIGN: Prospective observational study.

PARTICIPANTS: AIS patients discharged April 2016 to October 2017 from a safety-net hospital in South Carolina.

MAIN MEASURES: National Institutes of Health Stroke Scale (NIHSS) change at discharge (admission NIHSS score minus discharge NIHSS, continuous variable), 24h NIHSS change (attaining high improvement, admission NIHSS minus 24-h NIHSS being 75 th percentile or higher), door to neuroimaging (DTI) time, and IV alteplase receipt. NIHSS change was assessed within stroke severity groups, mild, moderate, and severe (admission NIHSS 0-5, 6-14, and $\geq 15$, respectively).

KEY RESULTS: Of 1168 patients, 838 were study-eligible (52\% male, 52.4\% Black, 72.2\% EMS arrivals, 56.6\% mild strokes). Severe and moderate stroke patients were more likely than mild stroke patients to use EMS (adjusted odds ratios, AOR [95\% CI] 11.7 [5.0, 27.4] and 4.0 [2.6, 6.3], respectively). EMS arrival was associated with shorter DTI time (adjusted difference - $88.4 \mathrm{~min}$ ) and higher likelihood of alteplase administration (AOR 5.3 [2.5, 11.4]), both key mediating variables in disability outcomes. High 24-h NIHSS improvement was more likely for EMS arrivals vs. other arrivals among moderate strokes (AOR 3.4 $[1.1,10.9]$ ) and severe strokes (AOR > 999). EMS arrivals had substantially higher NIHSS improvement at discharge within the severe stroke group (adjusted NIHSS change at discharge, 5.9 points higher, $p=0.01$ ). Alteplase recipients showed higher discharge NIHSS

Received November 18, 2019

Accepted August 5, 2020

Published online August 31, 2020 improvement than non-recipients (by 2.8 and 1.9 points among severe and moderate strokes, respectively; $p=$ $0.01,0.02)$.

CONCLUSIONS: The findings offer evidence for including stroke education as a standard of care in the primary care management of patients with stroke-risk comorbidities/ lifestyle in order to minimize post-stroke disability.

KEY WORDS: emergency medical services use; acute ischemic stroke; 24-h disability improvement; discharge disability outcome.

J Gen Intern Med 35(11):3173-80

DOI: $10.1007 / \mathrm{s} 11606-020-06114-4$

(c) Society of General Internal Medicine 2020

\section{INTRODUCTION}

Stroke affects over 795,000 Americans annually (38.9/ 100,000 population) causing 134,000 deaths (5\% of all deaths). ${ }^{1}$ The majority $(87 \%)$ are acute ischemic strokes (AIS) with high potential for disability mitigation with alteplase reperfusion (thrombolytic) treatment. ${ }^{2-4}$ Disabled stroke survivors account for over half of all long-term care costs; severely disabled survivors have fourfold and eightfold higher expenditures than the mildly disabled in the first and second years, respectively. ${ }^{3}$

Thrombolytic treatment significantly mitigates or reverses stroke disability; however, it must be administered within $4.5 \mathrm{~h}$ of stroke onset, ideally within $3 \mathrm{~h}^{5,6}$ Patients with suspected AIS who use emergency medical services (EMS) transport are more likely to arrive at hospital Emergency Departments (ED) within $2 \mathrm{~h}$ of stroke onset and to receive prompt intravenous alteplase. ${ }^{7}$ EMS use triggers certain actions: EMS staff notify the hospital ED of a brain attack patient en-route (BAT pre-notification), resulting in stroke team activation and clearing the neuroimaging suite to receive the patient. ${ }^{2,8}$ Even without BAT pre-notification, EMS 
arrival attracts immediate attention of the hospital emergency medical team, bypassing ED triage and wait times. ${ }^{9,10}$ Currently, about $59 \%$ of AIS patients arrive by EMS and about $30 \%$ get neuroimaging initiated within $3 \mathrm{~h}$ of stroke onset. ${ }^{11,12}$ Other transport arrivals may experience care delays due to ED crowding, uncommunicative aphasic or confused patients, and the absence of pain or severe discomfort among stroke patients. ${ }^{9}$ Many patients do not receive alteplase solely due to time considerations. ${ }^{9}$

Patient and bystander recognition of stroke symptoms and their EMS use decisions are critical for quick treatment initiation. AIS patient surveys in 1997, 2000, and 2003 showed that a third of stroke patients were unaware of any stroke symptom before their stroke, and three-fourths were unaware of the importance of quick treatment and EMS use for good outcomes. ${ }^{13-15}$ Our 2016-2017 survey of 108 hospitalized AIS patients (a subset of the current study sample), conducted within 2-5 days of their stroke, showed no change compared with the earlier 1997-2003 surveys: a third of our respondents were unaware of any stroke symptom before their stroke, and three-fourths were unaware of the importance of quick treatment and EMS use for good outcomes. ${ }^{16}$ Importantly, respondents' knowledge (or lack of knowledge) was reflected in their EMS use decisions. Two-thirds of patients in our survey patients reported not being educated by their physician about their stroke risk and about stroke symptoms despite an average prevalence of 2.4 stroke-risk factors among respondents. Nationally, EMS use by stroke patients has remained essentially stagnant, $59 \%$ in 2003 vs. $53 \%$ in $1997 .{ }^{12}$

The above findings suggest the need for prioritizing stroke education in the primary care management of patients with stroke risk factors. ${ }^{12}$ However, standard-of-care guidelines require evidence. There is no documented evidence of EMS association with stroke disability mitigation, although its association with quicker reperfusion treatment is documented. Establishing the disability outcome benefit of EMS is challenged by the confounding role of stroke severity at admission which impacts both patients' decision/compulsion to use EMS, and their residual disability. ${ }^{17}$ No clinical trial has randomized stroke patients to EMS and other transport, nor is it ethically defensible, given the established evidence of quicker thrombolytic treatment among EMS arrivals. Retrospective studies using claims data or stroke registry data are an option. However, claims data lack key data points, such as admission stroke severity and comfort care (hospice) assignment at admission. Pooled stroke registry data from hospitals have significant missing data on stroke severity (28\% missing in nationwide Get-With-The-Guidelines (GWTG) Stroke data for 2011-2014). ${ }^{11}$ This prospective study was conducted to assess the association of hospital arrival mode with clinical disability outcomes after accounting for stroke severity and other key factors influencing both reperfusion treatment decisions and disability recovery. Findings may have practice implications for secondary prevention of post-stroke disability.

\section{METHODS}

\section{Study Design and Data Source}

This prospective, observational cohort study assessed the associations of AIS patients' arrival mode with care processes and disability outcomes. The study setting was a Joint Commission-certified (JC, Oakbrook Terrace, IL) primary stroke center of a non-profit safety-net hospital affiliated with the University of South Carolina School of Medicine. The data source was the hospital's GWTG stroke registry database which is based on the American Heart Association's GWTG-Stroke Case Record Form. ${ }^{18}$ The registry database is routinely populated from patient electronic medical records (EMRs, hosted by Cerner, North Kansas City, MO) by trained registry staff. ${ }^{18}$ During the study period, registry staff extracted additional items from EMRs as required for the study. The study was approved by the hospital's Institutional Review Board (IRB).

\section{Selection of Participants}

Study inclusion criteria were patient discharged from April 1, 2016, to October 31, 2017, with a primary diagnosis of AIS (ICD-9CM 433.01, 433.11, 433.21, 433.31, 433.81, 433.91, $434.01,434.11,434.91$, or 436), cerebrovascular imaging evidence of AIS at admission, patient arrived directly at the study hospital (not a transfer patient), not assigned to comfort care/hospice at admission, and length of stay (LOS) less than 120 days (LOS $\geq 120$ days is administrative, pending placement in a long-term care facility). The sample selection criteria and resulting exclusions from total 1168 AIS discharges during the study period, and the final study-eligible sample, 838 patients, are shown in Figure 1 (605 EMS; 233 other transport).

\section{Outcomes and Analyses}

The primary outcomes of interest were care variables and patient outcome variables. Care variables were door to imaging (DTI) and door to needle (DTN) time in minutes, stroke onset to emergency department (ED) admission time, and receipt of IV alteplase. Times to events were calculated from GWTG registry-documented dates/times. Stroke onset to ED arrival time was ED arrival time minus last known well (arrival time was EMS arrival time or ED registration time for other arrivals). It was categorized as $\leq 3 \mathrm{~h}$ vs. otherwise (> $3 \mathrm{~h}$ or unknown). DTI was neuroimaging start time minus arrival time. For IV alteplase recipients, DTN was alteplase bolus time minus arrival time.

Patient outcome variables were (a) National Institutes of Health Stroke Scale (NIHSS) score improvement at discharge (admission NIHSS minus discharge NIHSS, continuous variable), (b) 24-h NIHSS change, high vs. otherwise (admission NIHSS minus 24-h NIHSS (measured at $24 \pm 8 \mathrm{~h}$ ). ${ }^{19}$ High improvement represented a score change of $\geq 75$ th percentile or 24-h NIHSS $0-2$, and (c) discharge disposition, died/ 


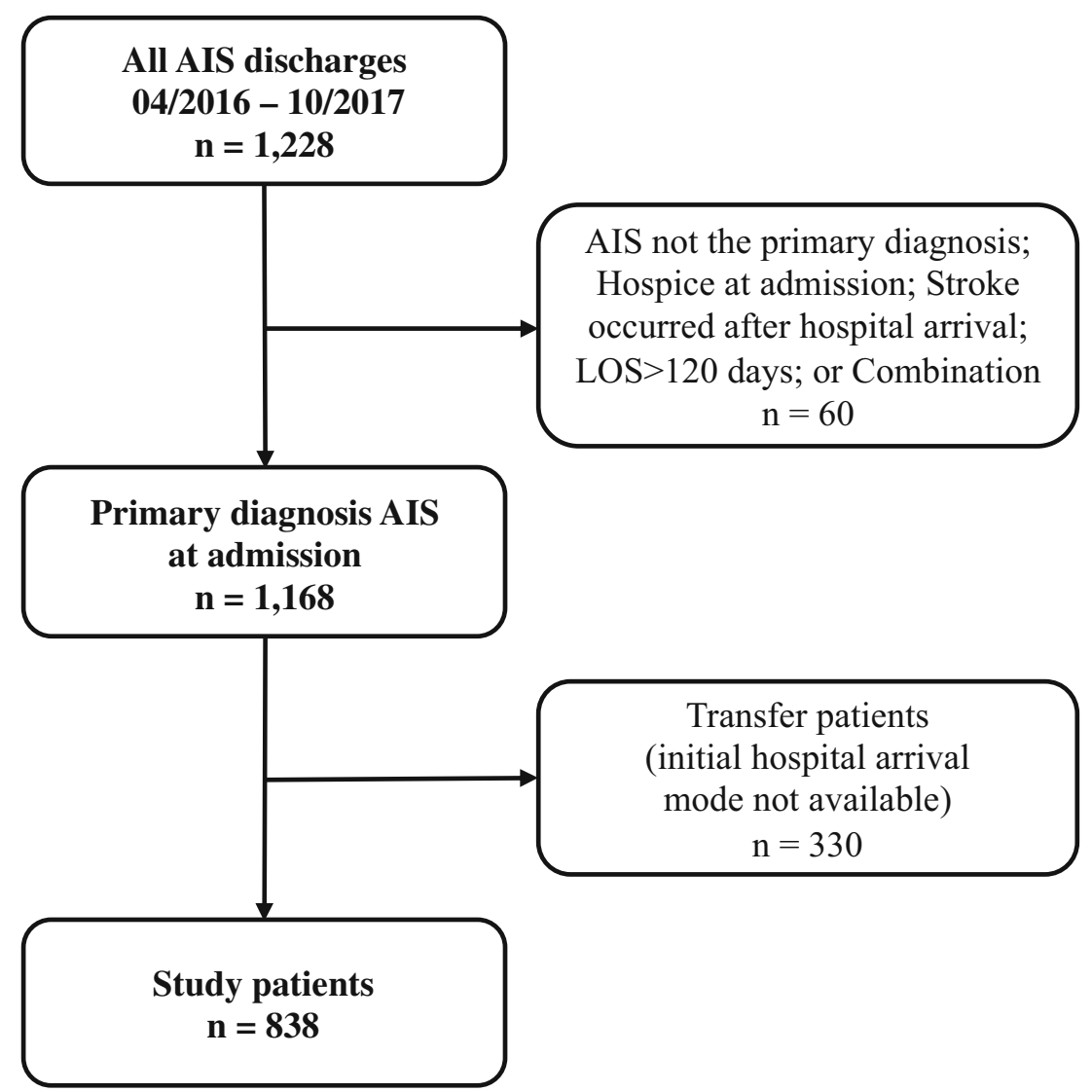

Figure 1 Study-eligible patients after applying exclusion criteria (AIS, acute ischemic strokes; LOS, length of stay (days)).

assigned to hospice vs. otherwise. ${ }^{20}$ NIHSS at $24 \mathrm{~h}$ accurately predicts the 90-day modified Rankin Score (mRS) disability status. ${ }^{19}$ Discharge NIHSS was captured using the following hierarchy: last NIHSS within $24 \mathrm{~h}$ of discharge; if unavailable, last NIHSS on or after the 7th day of admission; last NIHSS 0 2 with the previous NIHSS within \pm 2 points of last NIHSS. By protocol, NIHSS assessment is stopped on achieving stable NIHSS of $0-2$. NIHSS change was studied within stroke severity categories (mild: admission NIHSS $0-5$, moderate: $6-14$, severe: $15-37){ }^{21}$

The key independent variable was arrival mode, EMS vs. other transport. BAT pre-notification was not mandatory at the study hospital; data are not available. The key covariate of interest was admission stroke severity category. ${ }^{21}$ Other covariates were demographic characteristics (age in years, sex, race [Black, White/others; too few other race]), endovascular therapy (EVT, Yes/No), onset to admission time $(0-3 \mathrm{~h},>3-4.5 \mathrm{~h}$, $>4.5 \mathrm{~h}$, unknown), history of GWTG stroke-risk comorbidities (each included in the final models if statistically significant), previous stroke, previous transient ischemic attack (TIA), family history of stroke, smoking (current smoker vs. otherwise), history of drug/alcohol abuse, and absolute or relative contraindication to alteplase (Yes/No), and receipt of inpatient rehab services. GWTG comorbidities are atrial fibrillation, coronary artery disease/prior myocardial infarction, carotid stenosis, peripheral vascular disease, depression, diabetes mellitus, hypertension, dyslipidemia, heart failure, migraine, obesity, renal insufficiency, and sleep apnea. Other variables were insurance type (uninsured, Medicaid, other insurance) and ED arrival shift (weekday morning: Mon-Fri 7:00 AM to 1:00 PM; weekday afternoon: Monday-Friday 1:00 PM to 9:00 PM; night/weekend: Monday-Thursday nights, Friday 9:01 PM to Monday 6:59 AM).

Univariate comparisons of EMS and other arrivals were performed using chi-square and $t$ tests for categorical and continuous variables, respectively. Factors associated with the likelihood of EMS use were studied using logistic regression analysis. Associations of arrival mode with DTI time, DTN time, alteplase receipt, 24-h and discharge NIHSS change, and discharge disposition were studied using logistic regression analysis for binary outcomes and linear regression analysis for continuous variables. Linear regression was used to study the association of arrival mode with discharge NIHSS change after verifying normality of discharge NIHSS change using the Kolmogorov-Smirnov test. All clinical variables (e.g., GWTG comorbidity, endovascular treatment, receipt of inpatient rehab services) and care logistic variables (e.g. admission shift) that could potentially influence the outcome of interest were included in the respective regression models. Starting with the full model, the backward variable selection strategy with stepwise manual variable removal was used. The final models retained statistically significant covariates, the demographic variables, and key independent variables. Interactions of arrival mode and stroke severity in impacting the 
outcomes of interest were assessed. If significant, the adjusted difference in the outcome variable between EMS and other arrivals (or odds ratio) was generated within each stroke severity group, mild, moderate, and severe. Association of IV alteplase with discharge NIHSS change was assessed with a similar strategy. Statistical analyses were performed in SAS, version 9.4 (SAS, Cary, NC), using $p<0.05$ for statistical significance.

\section{Sample Size and Power Calculation}

Sample size and power calculations were based on the study hospital's 2015 proportion of EMS arrival rate $(50 \%)$ and alteplase administration rate (3.5\%). At $80 \%$ power, a sample of 1080 patients was deemed adequate to detect odds ratios of 1.75 for EMS vs. other arrivals for binary outcomes and to study continuous dependent variables.

\section{RESULTS}

\section{Study Patients}

Of 838 patients, $52 \%$ were male and $52.4 \%$ were Black. Their mean age was 68.3 years (SD 13.6), $72.2 \%$ were EMS arrivals, and $56.4 \%$ had mild strokes with a mean of 3.7 GWTG stroke risk factors per patient (SD 1.9) (Table 1). The mean admission NIHSS (available for $96.7 \%$ of patients) was 6.8 (SD 7.3); median NIHSS 4.0. EMS arrivals were older and had more severe stroke (mean admission NIHSS 8.3 vs. other arrivals $2.9, p<0.0001$ ).

Table 2 presents patient care and outcomes by arrival mode. Of the total patients, 92 were ineligible for discharge NIHSS (31 expired, 61 hospice care), and of 746 eligible, 91.4\% (682 patients) had discharge NIHSS data available (not shown in the table). Of the total patients, 114 (13.6\%) received alteplase. The median DTI time was $49 \mathrm{~min}$, and among alteplase recipients, $17.5 \mathrm{~min}$. The median DTN time for alteplase recipients was $38 \mathrm{~min}$ (IQR 26-61). EMS arrivals had shorter DTI time than other transport, and significantly greater percentages were admitted within $3 \mathrm{~h}$ of stroke onset (35\% vs. $12 \% ; p<0.0001)$ and received IV alteplase $(17.4 \%$ vs. $3.9 \%$; $p<0.0001)$. Most alteplase recipients (105 of 114) and EVT recipients (51 of 56) were EMS arrivals. The 75th percentile of 24-h NIHSS change for the sample was 2 NIHSS points. Significantly greater proportion of EMS arrivals achieved this level of improvement or higher (31.6\% vs. $22.8 \%, p=0.006$ ). EMS arrivals had greater discharge NIHSS improvement (mean change 1.8 vs. $0.7 ; p=0.0015$ ), higher percentage of death/hospice outcome $(14.7 \%$ vs. $1.3 \% ; p<0.0001)$, and longer LOS (11.1 days vs. 6.9, $p=0.009$ ), compared with other transport.

\section{Factors Associated with EMS Use}

Stroke severity was the strongest predictor of EMS use (Table 3). Severe stroke patients had much higher odds of
EMS arrival relative to mild stroke (adjusted odds ratio (AOR [95\% CI]), 11.7 [5.0, 27.4], as also moderate stroke patients relative to mild stroke, AOR $4.0[2.6,6.3]$ ), indicating that arrival mode impacts on outcomes should be studied within severity groups in regression analyses.

\section{EMS Associations with Care Processes}

Table 4 presents the adjusted associations of arrival mode with patient care processes and discharge outcomes. EMS arrivals were more likely to be admitted within $3 \mathrm{~h}$ of stroke onset (AOR $4.2[2.6,6.7]$ ) after accounting for demographics, admission stroke severity, and pre-admission clinical factors. EMS arrivals were also more likely to receive IV alteplase (AOR 5.3, [2.5, 11.4]) and had shorter covariate-adjusted DTI time than other transport patients $(-88.4 \mathrm{~min})$, but similar DTN time $(p=0.39){ }^{22}$

\section{EMS Use and Patient Outcomes}

Table 4 shows that EMS arrivals had higher adjusted odds of achieving high 24-h NIHSS change relative to other transport within the moderate and severe stroke groups. Among moderate strokes, AOR was 3.4 [1.05, 10.9], and among severe strokes, AOR was > 999 (all 26 severe stroke patients with high 24-h improvement were EMS arrivals). Among mild strokes, arrival mode was not significantly associated with 24-h NIHSS change. Considering NIHSS change at discharge, among severe stroke patients, EMS arrivals had a covariateadjusted mean NIHSS improvement of 13.0 points vs. 7.1 among other arrivals, a 5.9-point difference $(p<0.01)$. Differences within the mild and moderate stroke groups were not statistically significant. Considering discharge disposition, after adjustment for severity and other covariates, arrival mode was not associated with death/hospice outcome in any severity group. Adjusted LOS was significantly lower for EMS arrivals than other transport within mild and moderate stroke groups ( $p$ $=0.002$ and 0.003 ), but not among the severe stroke group. However, the magnitude of differences was small, $<1$ day.

\section{Alteplase and Discharge Disability Outcome}

Table 5 shows that within the moderate and severe stroke groups, IV alteplase was associated with higher NIHSS score improvement at discharge compared with no-alteplase (adjusted difference of 1.9 and 2.8 points respectively within the moderate and severe stroke groups, $p=0.02$ and 0.01 ). Alteplase was not associated with discharge NIHSS improvement among the mild stroke group.

\section{DISCUSSION}

This study reports a statistically significant and clinically meaningful magnitude of association between hospital arrival mode and disability outcomes within severe and moderate stroke groups. We used a prospective, observational study 
Table 1 Characteristics of Acute Ischemic Stroke (AIS) Patients at Admission, Classified by Hospital Arrival Mode

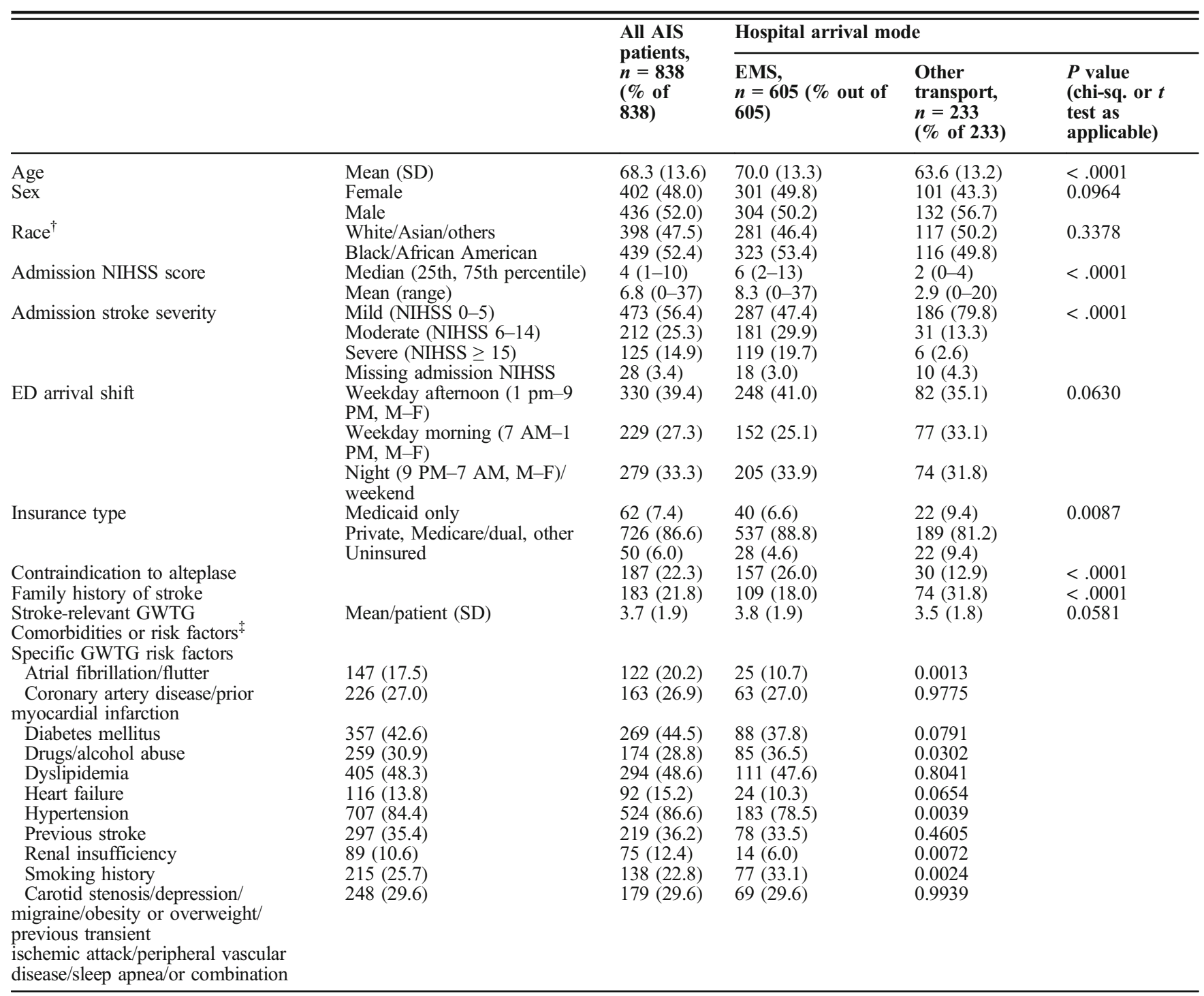

AIS acute ischemic stroke, EMS emergency medical services, NIHSS National Institutes of Health Stroke Scale, ED emergency department +1 patient had missing data on race

$\neq$ fWTG (Get With The Guidelines)-stroke comorbidities: atrial fibrillation/flutter, coronary artery disease/prior myocardial infarction, carotid stenosis, depression, diabetes mellitus, drugs/alcohol abuse, dyslipidemia, heart failure, hypertension, migraine, obesity/overweight, previous stroke, previous transient ischemic attack, peripheral vascular disease, renal insufficiency, sleep apnea, and smoking history

design with minimal missing data on stroke severity. EMS arrivals with severe stroke experienced 5.9 NIHSS points higher neurological improvement at discharge than other arrivals after adjusting for demographic, comorbidity, and care variables. This magnitude of effect has potentially lifelong implications for independence in daily living (ADL), productivity, quality of life, and long-term care costs. EMS users among moderate and severe stroke groups also achieved higher disability reduction at $24 \mathrm{~h}$, a measure that accurately predicts post-discharge disability (90-day mRS). ${ }^{19}$ Absence of significant differences in 24-h and discharge NIHSS change within the mild stroke group is partly attributable to the "floor" effect (admission NIHSS of 0-2 has little to no room for improvement).
The observed associations of EMS with neurological disability outcomes are validated by its associations with consecutive events in the chain of AIS care. EMS arrivals were four times as likely as other arrivals to reach the hospital within the optimum alteplase window, received neuroimaging within half the time that it took for other arrivals, and were more likely to receive IV alteplase (fivefold odds). The latter findings show the mechanism by which EMS users achieved substantially higher NIHSS score improvement at $24 \mathrm{~h}$ and at discharge and support the conclusion that EMS arrival indeed contributed to the observed functional outcome benefits. A meta-analyses involving multiple studies reported that every $15 \mathrm{~min}$ saved in starting alteplase treatment reduces residual disability among stroke survivors by $4 \% .^{6,20,23}$ Our 
Table 2 Bivariate Distribution of Arrival Mode with Care Receipt/Timeliness, and Patient Outcomes

\begin{tabular}{|c|c|c|c|c|c|}
\hline & & \multirow{2}{*}{$\begin{array}{l}\text { All AIS patients, } n= \\
838(\% \text { of } 838)\end{array}$} & \multicolumn{3}{|c|}{ Hospital arrival mode } \\
\hline & & & $\begin{array}{l}\text { EMS, } n=605 \\
(\% \text { of } 605)\end{array}$ & $\begin{array}{l}\text { Other transport, } n= \\
233(\% \text { of } 233)\end{array}$ & $\begin{array}{l}P \text { value (chi-sq. or } t \\
\text { test as applicable) }\end{array}$ \\
\hline \multirow[t]{2}{*}{ Door to imaging time $(\mathrm{min})$} & $\begin{array}{l}\text { Median (25th, } 75 \text { th } \\
\text { percentile)-all patients }\end{array}$ & $49(20-109)$ & $36(17-81)$ & $93(47-229)$ & $<.0001$ \\
\hline & $\begin{array}{l}\text { IV TPA patients (114 } \\
\text { patients) }\end{array}$ & $17.5(10-25)$ & $17(10-24)$ & $23(15-41)$ & 0.0881 \\
\hline Door to needle time (min) & $\begin{array}{l}\text { Median }(25 \text { th, } 75 \text { th } \\
\text { percentile) }\end{array}$ & $38(26-61)$ & $38(26-61)$ & $42(34-56)$ & 0.1073 \\
\hline \multicolumn{2}{|c|}{ Intravenous alteplase at study hospital } & $114(13.6)$ & $105(17.4)$ & $9(3.9)$ & $<.0001$ \\
\hline \multicolumn{2}{|c|}{ Endovascular treatment (EVT) } & $56(6.7)$ & $51(8.4)$ & $5(2.2)$ & 0.0011 \\
\hline Stroke onset to adm. time & $0-3 \mathrm{~h}$ & $240(28.6)$ & $213(35.2)$ & $27(11.6)$ & $<.0001$ \\
\hline \multirow[t]{3}{*}{ (Adm.- last known well) } & $>3-4.5 \mathrm{~h}$ & $76(9.1)$ & $59(9.8)$ & $17(7.3)$ & \\
\hline & $>4.5 \mathrm{~h}$ & $288(34.4)$ & $193(31.9)$ & $95(40.8)$ & \\
\hline & Unknown & $234(27.9)$ & $140(23.1)$ & $94(40.3)$ & \\
\hline \multirow[t]{3}{*}{ 24-h NIHSS change } & $\begin{array}{l}\text { High }(\geq 75 \text { th or } 24 \text { - } \\
\text { h NIHSS }<=2)\end{array}$ & $244(29.1)$ & $191(31.6)^{\dagger}$ & $53(22.8)^{\dagger}$ & $0.0060^{*}$ \\
\hline & $\begin{array}{l}\text { Not high }(<75 \text { th } \\
\text { percentile })\end{array}$ & $527(62.9)$ & $362(59.8)$ & $165(70.8)$ & \\
\hline & Missing* & $67(8.0)$ & $52(8.6)$ & $15(6.4)$ & \\
\hline $\begin{array}{l}\text { NIHSS change Adm. to } \\
\text { Disch }^{\S}\end{array}$ & Mean (SD) & $1.5(6.4)$ & $1.8(7.3)$ & $0.7(3.4)$ & 0.0015 \\
\hline \multirow[t]{4}{*}{ Discharge disposition } & Expired & $31(3.7)$ & $29(4.8)$ & $2(0.9)$ & $<.0001$ \\
\hline & Hospice & $61(7.3)$ & $60(9.9)$ & $1(0.4)$ & \\
\hline & Discharged to home & $410(48.9)$ & $235(38.8)$ & $175(75.1)$ & \\
\hline & $\begin{array}{l}\text { Discharged to other } \\
\text { facility }\end{array}$ & $336(40.1)$ & $281(46.5)$ & $55(23.6)$ & \\
\hline Length of stay (days) & Mean (SD) & $9.9(17.5)$ & $11.1(18.8)$ & $6.9(13.2)$ & 0.0099 \\
\hline
\end{tabular}

EMS emergency medical services, Adm admission, NIHSS National Institutes of Health Stroke Scale

*26 patients had missing admission NIHSS, and 41 patients missing 24-h NIHSS, total 67 patients missing 24-h NIHSS change

+Number of patients in this arrival mode category who were at or above the 75th percentile level for the full sample

$\$ p=0.0060$ after excluding missing from chi-square analysis

\$NIHSS change at discharge available for 681 patients. After excluding 92 expired/hospice patients, 22 of the remaining had missing admission NIHSS, 42 had missing discharge NIHSS

study documents the positive effects of EMS transportation on alteplase receipt, other intermediate steps in the chain of stroke care, and ultimately, neurological disability outcomes.

We found no difference in death/hospice outcome among EMS vs. other transport after accounting for admission stroke

Table 3 Factors Associated with EMS Arrival: Multiple Regression Analysis Results * $(n=809$ Patients $)$

\begin{tabular}{ll}
\hline \hline & $\begin{array}{l}\text { Adjusted odds ratio (95\% } \\
\text { CI), } \boldsymbol{p} \text { value }\end{array}$ \\
\hline $\begin{array}{l}\text { Admission stroke severity } \\
\text { Moderate (vs. mild) }\end{array}$ & $4.0(2.6,6.3),<.0001$ \\
$\begin{array}{l}\text { Severe (vs. mild) } \\
\text { History of atrial fibrillation: yes (vs. }\end{array}$ & $11.7(5.0,27.4),<.0001$ \\
no) & $0.7(0.4,1.0), 0.0438$ \\
History of CAD or prior MI: yes (vs. & 0.0375 \\
no) & $2.1(1.0,4.2), 0.0389$ \\
Depression: yes (vs. no) & $0.5(0.4,0.8), 0.0008$ \\
$\begin{array}{l}\text { Family history of stroke: yes (vs. no) } \\
\text { Age }\end{array}$ & $1.0(1.0,1.1),<.0001$ \\
Female (vs. male) & $1.1(0.8,1.5), 0.6997$ \\
Black/African American (vs. White/ & $1.6(1.1,2.2), 0.0133$ \\
Asian/other) & \\
\hline
\end{tabular}

EMS emergency medical services, CAD coronary artery disease, MI myocardial infarction

Analytic sample is less than the full sample due to missing data on admission NIHSS score and race

tNo other potentially influential variable was significant, i.e., ED arrival shift, health insurance status, and GWTG comorbidities, not included in final model severity and comorbidities. In contrast, the unadjusted comparison shows higher rate of death/hospice outcome among EMS arrivals which is attributable to more severe strokes among EMS arrivals as observed in Table 1. Together, the findings support a true disability reduction benefit of EMS, and that the observed benefit is not an artifact due to survival bias. Our findings regarding EMS impacts on the processes of care (DTI, DTN, admission within the alteplase window, and alteplase receipt) are similar to those reported in the documented literature. ${ }^{8,10}$

This study contributes to the general internal medicine literature by offering evidence of substantial disability mitigation by EMS use in a community-based patient cohort managed at a primary stroke center. Our findings may be widely applicable to stroke patients. Our cohort stroke severity is similar to the 2011-2014 nationwide GWTG stroke cohort (median admission NIHSS 4.0). ${ }^{11}$ The nationwide EMS rate of 59\% across all AIS patients is somewhat higher than our cohort rate of $52 \%{ }^{12}$ Overall, the observed associations between EMS use and outcomes have evidentiary value for primary care practice.

Prompt EMS use by stroke patients could be facilitated by systematic stroke education of patients with chronic medical conditions, smoking, and other lifestyle factors in primary care settings. Study patients had an average of 3.7 stroke-risk comorbidities/lifestyle factors per patient including widely 
Table 4 Adjusted Estimates for Receipt/Timeliness of Care and Clinical Outcomes, EMS vs. Other Transport Arrivals (Numeric Difference or Odds Ratio as Applicable)

\begin{tabular}{|c|c|c|}
\hline \multirow{2}{*}{$\begin{array}{l}\text { Outcome modeled in } \\
\text { each regression* }\end{array}$} & \multicolumn{2}{|c|}{ EMS vs. other transport ${ }^{\dagger}$} \\
\hline & $\begin{array}{l}\text { Adj. mean } \\
\text { difference (SE), } p \\
\text { value }\end{array}$ & $\begin{array}{l}\text { Adj. odds ratio } \\
\text { (95\% CI), } p \\
\text { value }\end{array}$ \\
\hline \multicolumn{3}{|l|}{ Process of care } \\
\hline $\begin{array}{l}\text { Door to imaging time } \\
\text { (min) }\end{array}$ & $\begin{array}{l}-88.4(12.4),< \\
.0001\end{array}$ & - \\
\hline $\begin{array}{l}\text { Door to needle time } \\
\text { (min) }\end{array}$ & -8.5 (9.8), 0.3874 & - \\
\hline $\begin{array}{l}\text { Admitted within } 3 \mathrm{~h} \text { of } \\
\text { stroke onset }\end{array}$ & - & $\begin{array}{l}4.2(2.6,6.7),< \\
.0001\end{array}$ \\
\hline $\begin{array}{l}\text { Likelihood of IV } \\
\text { alteplase }\end{array}$ & - & $\begin{array}{l}5.3(2.5,11.4),< \\
.0001\end{array}$ \\
\hline $\begin{array}{l}\text { Patient outcome } \\
\text { 24-h NIHSS change } \\
\text { (high vs. not high) }\end{array}$ & & \\
\hline Mild & - & $\begin{array}{l}0.9(0.6,1.4) \\
0.7799\end{array}$ \\
\hline Moderate & - & $\begin{array}{l}3.4(1.1,10.9), \\
0.042\end{array}$ \\
\hline Severe & - & $>999.999^{\S}$ \\
\hline \multicolumn{3}{|l|}{$\begin{array}{l}\text { NIHSS change at } \\
\text { discharge (adm.--disch. } \\
\text { NIHSS) }\end{array}$} \\
\hline $\begin{array}{l}\text { Mild (EMS = 2.4; } \\
\text { other }=2.3)\end{array}$ & $0.1,0.7753$ & - \\
\hline $\begin{array}{l}\text { Moderate }(\text { EMS }=5.3 \text {; } \\
\text { other }=5.5)\end{array}$ & $-0.2,0.8314$ & - \\
\hline $\begin{array}{l}\text { Severe }(\mathrm{EMS}=13.0 \text {; } \\
\text { other }=7.1)\end{array}$ & $5.9,0.0046$ & - \\
\hline \multicolumn{3}{|l|}{$\begin{array}{l}\text { Discharge disposition: } \\
\text { expired/hospice vs. other }\end{array}$} \\
\hline Mild & - & $\begin{array}{l}5.5(0.7,45.0) \\
0.1095\end{array}$ \\
\hline Moderate & - & $\begin{array}{l}4.0(0.5,31.4), \\
0.1859\end{array}$ \\
\hline Severe & - & $\begin{array}{l}2.4(0.3,22.4) \\
0.4447\end{array}$ \\
\hline Length of stay (days) & & \\
\hline $\begin{array}{l}\text { Mild }(\text { EMS = 2.5; } \\
\text { other }=2.3)\end{array}$ & $0.2,0.0016$ & - \\
\hline $\begin{array}{l}\text { Moderate }(\mathrm{EMS}=3.2 ; \\
\text { other }=2.7)\end{array}$ & $0.5,0.0032$ & - \\
\hline $\begin{array}{l}\text { Severe (EMS = 3.3; } \\
\text { other }=3.8)\end{array}$ & $-0.5,0.2037$ & - \\
\hline
\end{tabular}

EMS emergency medical services, NIHSS National Institutes of Health Stroke Scale, SE standard error

*Analytic samples used for regressions vary from the full sample based on eligibility for the outcome and/or missing data on the dependent/key independent variables. Analytic samples in the order of models presented above were 799, 114, 809, 809, 769, 681, 819, and 809 respectively. Large sample drop for NIHSS change at discharge is due to 92 expired or hospice outcomes, therefore ineligible for discharge NIHSS assessment. Sample for door to needle time is 114 patients who received alteplase

†All models adjusted for age, sex, race, stroke severity; each model also adjusted for other independent variables relevant for the outcome $\$ 24-h$ NIHSS change, high if admission NIHSS minus 24-h NIHSS value was at or higher than the 75th percentile value for the full sample, or absolute NIHSS, $0-2$ at $24 \mathrm{~h}$

$\$$ Model did not produce an interpretable odds ratio estimate for severe stroke because all 26 patients with high 24-h NHISS change were EMS arrivals

prevalent conditions such as diabetes, hypertension, smoking, and ischemic heart disease. Yet, a third of them had no knowledge of any stroke symptom and three-fourths were unaware of the importance of quick treatment and EMS
Table 5 Adjusted Mean NIHSS Change at Discharge, Alteplase vs. No-Alteplase, Within Each Stroke Severity Category $(n=681)$

\begin{tabular}{lllll}
\hline \hline & \multicolumn{2}{l}{$\begin{array}{l}\text { Least square regression-adjusted mean } \\
\text { NIHSS change }\end{array}$} & P value \\
\cline { 2 - 4 } & Alteplase & No alteplase & Difference & \\
\hline Mild & 2.1 & 2.1 & 0 & 0.9920 \\
Moderate & 6.5 & 4.6 & 1.9 & 0.0215 \\
Severe & 14.3 & 11.5 & 2.8 & 0.0133 \\
\hline
\end{tabular}

NIHSS National Institutes of Health Stroke Scale

tAdjusted for demographic, GWTG comorbidities, endovascular treatment, rehab care receipt, and other relevant variables

use. ${ }^{16}$ Together, our two studies may present an evidence base for incorporating stroke education as a standard of care in the primary care management of patients with stroke-risk factors. Additional efforts beyond medical professional efforts are essential to minimize post-stroke disability - population-wide education to increase general awareness of stroke, regulatory and insurer policies to mitigate EMS costs, measures to improve EMS access for rural populations, and stroke education of frontline ED staff including patient registration staff.

\section{STUDY LIMITATIONS}

Single-center study limitation is partly mitigated by similarity of cohort composition to multicenter GWTG cohorts (similar median admission NIHSS). A singlecenter study, however, facilitated maximum data extraction on admission and discharge NIHSS by EMR text search $(<10 \%$ missing data vs. $28 \%$ in multicenter GWTG studies). ${ }^{11}$ The observational study design is another limitation. However, randomized clinical trials are not feasible for ethical reasons. Other limitations are the absence of data on BAT pre-notification and 90-day mRS outcomes. The latter may be mitigated by $24-\mathrm{h}$ and discharge NIHSS which correlate with 90-day mRS. The validity of the evidence generated is reinforced by the study strengths: prospective design, minimal missing data, and adjustment for most covariates known to influence functional outcomes (age, race, stroke severity, GWTG risk factors, and alteplase contraindication).

Acknowledgments: The authors are grateful to Ms. Iris Smith, Director of Rehabilitation Services, Prisma Health (formerly Palmetto Health Richland hospital), and the Prisma Health Stroke Unit staff, Amanda Cotter, Andrea Griffin, Karen Cartrett, and Taylor Kizer, for advisory input regarding the GWTG data elements and logistical organization of the study, extraction of additional data from patient EMRs, preparing the de-identified study database out of GWTG data, and coordination assistance during the study. We appreciate the helpful comments on an earlier version of the manuscript by Marquita Decker-Palmer, $M D, P h D$, of Genentech, Inc.

Corresponding Author: Sudha Xirasagar, MBBS, PhD; Department of Health Services Policy and Management, Arnold School of Public Health, University of South Carolina, Columbia, SC, USA (e-mail: sxirasagar@sc.edu). 
Funding Information This study was supported by a grant from $F$. Hoffmann-La Roche, Ltd., and Genentech, Inc. All funds were used towards study staff time-effort and data collection costs.

\section{Compliance with Ethical Standards:}

The study was approved by the hospital's Institutional Review Board (IRB).

Conflict of Interest: Authors' time-effort for the study were partially funded by a grant from Genentech, Inc. to the University of South Carolina Research Foundation, a registered 501 (c) non-profit institution. Genentech, Inc. is the sole manufacturer of alteplase, the key thrombolytic drug used in ischemic stroke. It may be noted, however, that the study topic and research questions are not related in any way to alteplase drug effectiveness, clinical indications or any commercially relevant aspect. None of the authors has any conflict of interest. All authors received financial support for their time-effort via the university foundation as itemized below.

SX: SX received a part of her summer salary for 2 years from the grant. The grant partially supported her time-effort on the project, the remaining time-effort being covered by her 9-month institutional salary from the University of South Carolina. SX's travel to the American College of Emergency Physicians Annual Meeting 2018 to present the study findings was reimbursed from the grant. SX has not received any other financial or in-kind support/revenue from Genentech, Inc. or its allied subsidiaries, nor has any financial interest in Genentech or allied industry.

MT, YW, and JZ: MT's salary as a graduate research assistant and later as a post-doctoral fellow was partly covered by the Genentech, Inc. grant. JZ and YW were compensated as graduate research assistants by the University of South Carolina at standard rates for graduate assistants. None of them has any other conflicts of interest relative to industry including Genentech.

$\mathrm{KH}$ : KH was compensated as a consultant by the university from grant funds. His compensation amounted to $<10 \%$ of his full-time job salary as Senior Epidemiologist of the South Carolina Department of Health and Environmental Control. $\mathrm{KH}$ was also compensated from the grant for travel expenses to present the findings at the American College of Emergency Physicians Annual Meeting 2018.

JWH: JWH received a small percentage of his 3-month summer salary for 2 years from the grant towards part of his time-effort devoted to the project.

DH: DH is a former employee of Genentech, Inc. and was the scientific liaison for the project. She is since no longer with Genentech, Inc. ECJ: ECJ was compensated a small consulting fee from the grant via the university foundation towards his time-effort, the total being $<1 \%$ of the permitted National Institutes of Health base salary for federal grants.

SS: SS received 2\% salary support for his time-effort on the project for 2 years.

\section{REFERENCES}

1. Mozaffarian D, Benjamin EJ, Go AS, et al. Executive summary: heart disease and stroke statistics—2015 update: a report from the American Heart Association. Circulation. 2015;131(4):434-441.

2. Lin CB, Peterson ED, Smith EE, et al. Emergency medical service hospital prenotification is associated with improved evaluation and treatment of acute ischemic stroke. Circulation: Cardiovascular quality and outcomes. 2012;5(4):514-522.

3. Lekander I, Willers C, Von Euler M, et al. Relationship between functional disability and costs one and two years post stroke. PloS one. 2017;12(4):e0174861

4. Lindgren P, Glader E-L, Jönsson B. Utility loss and indirect costs after stroke in Sweden. European Journal of Cardiovascular Prevention \& Rehabilitation. 2008;15(2):230-233.

5. Powers WJ, Rabinstein AA, Ackerson T, et al. 2018 guidelines for the early management of patients with acute ischemic stroke: a guideline for healthcare professionals from the American Heart Association/American Stroke Association. Stroke. 2018;49(3):e46-e99.
6. Saver JL, Fonarow GC, Smith EE, et al. Time to treatment with intravenous tissue plasminogen activator and outcome from acute ischemic stroke. Jama. 2013;309(23):2480-2488.

7. Tong $\mathbf{D}, \mathbf{R e e v e s} \mathbf{M J}$, Hernandez AF, et al. Times from symptom onset to hospital arrival in the Get with the Guidelines-Stroke Program 2002 to 2009: temporal trends and implications. Stroke. 2012;43(7):1912-1917.

8. Patel MD, Rose KM, O'Brien EC, Rosamond WD. Prehospital notification by emergency medical services reduces delays in stroke evaluation: findings from the North Carolina stroke care collaborative. Stroke. 2011;42(8):2263-2268.

9. Romano JG, Muller N, Merino JG, Forteza AM, Koch S, Rabinstein AA. In-hospital delays to stroke thrombolysis: paradoxical effect of early arrival. Neurological research. 2007;29(7):664-666.

10. Fassbender K, Balucani C, Walter S, Levine SR, Haass A, Grotta J. Streamlining of prehospital stroke management: the golden hour. The Lancet Neurology. 2013;12(6):585-596.

11. Mochari-Greenberger H, Xian Y, Hellkamp AS, et al. Racial/ethnic and sex differences in emergency medical services transport among hospitalized US stroke patients: analysis of the National Get With The GuidelinesStroke Registry. Journal of the American Heart Association. 2015;4(8):e002099.

12. Mohammad YM. Mode of arrival to the emergency department of stroke patients in the United States. Journal of vascular and interventional neurology. 2008;1(3):83.

13. Schroeder EB, Rosamond WD, Morris DL, Evenson KR, Hinn AR. Determinants of use of emergency medical services in a population with stroke symptoms: the Second Delay in Accessing Stroke Healthcare (DASH II) Study. Stroke. 2000;31(11):2591-2596.

14. Kleindorfer D, Khoury J, Broderick JP, et al. Temporal trends in public awareness of stroke: warning signs, risk factors, and treatment. Stroke. 2009;40(7):2502-2506.

15. Meurer WJ, Levine DA, Kerber KA, et al. Neighborhood Influences on Emergency Medical Services Use for Acute Stroke: A Population-Based Cross-sectional Study. Annals of emergency medicine. 2016;67(3):341348.e344.

16. Xirasagar S, Tsai MH, Heidari K, Hardin JW, Wu Y, Wronski R, Hurley D, Jauch EC, Sen S. Why Acute Ischemic Stroke Patients in the United States Use or Do Not Use Emergency Medical Services Transport? Findings of an Inpatient Survey. BMC Health Services Research, 2019: In press.

17. Williams LS, Bruno A, Rouch D, Marriott DJ, MAS. Stroke patients' knowledge of stroke: influence on time to presentation. Stroke. 1997;28(5):912-915.

18. American Heart Association 2016. Get With The Guidelines Case Record Form. https://www.heart.org/-/media/files/professional/quality-improvement/get-with-the-guidelines/get-with-the-guidelines-stroke/caserecordform91718.pdf?la=en\&hash=48474515D0DF857F0E50540B0306AFCF8C21D2CF. Accessed Mar 3, 2020 and April 20, 2020

19. Rangaraju S, Frankel M, Jovin TG. Prognostic value of the 24-hour neurological examination in anterior circulation ischemic stroke: a post hoc analysis of two randomized controlled stroke trials. Interventional neurology. 2015;4(3-4):120-129.

20. Lees KR, Bluhmki E, Von Kummer R, et al. Time to treatment with intravenous alteplase and outcome in stroke: an updated pooled analysis of ECASS, ATLANTIS, NINDS, and EPITHET trials. The Lancet. 2010;375(9727):1695-1703.

21. Govan $\mathbf{L}$, Langhorne $\mathbf{P}$, Weir $\mathbf{C J}$. Categorizing stroke prognosis using different stroke scales. Stroke. 2009;40(10):3396-3399.

22. Jauch EC, Saver JL, Adams Jr HP, et al. Guidelines for the early management of patients with acute ischemic stroke: a guideline for healthcare professionals from the American Heart Association/American Stroke Association. Stroke. 2013;44(3):870-947.

23. Emberson J, Lees KR, Lyden $\mathbf{P}$, et al. Effect of treatment delay, age, and stroke severity on the effects of intravenous thrombolysis with alteplase for acute ischaemic stroke: a meta-analysis of individual patient data from randomised trials. The Lancet. 2014;384(9958):1929-1935.

Publisher's Note: Springer Nature remains neutral with regard to jurisdictional claims in published maps and institutional affiliations. 